\title{
Measurement of Cellular Immunity to Influenza Vaccination in Rheumatoid Arthritis; Comparison of Three Assays
}

Noa Madar-Balakirski ${ }^{1}$, Uri Arad ${ }^{1}$, Sharon Amir ${ }^{1}$, Michal Mandelboim $^{2}$, Ella Mendelson ${ }^{2}$, Dan Caspi ${ }^{1}$ and Ori Elkayam $^{1^{*}}$

${ }^{1}$ Department of Rheumatology, Tel Aviv Sourasky Medical Center, Tel Hashomer, Israel

${ }^{2}$ Central Virology Laboratory, Sheba Medical Center, Tel Hashomer, Israel

"Corresponding author: Ori Elkayam, Department of Rheumatology, Tel-Aviv Sourasky Medical Center 6 Weizmann St. Tel-Aviv 64239, Israel, Tel: +972-3-6973668; Fax: +972-3-6974577; E-mail:

Received date: 03 January 2015; Accepted date: 23 March 2015; Published date: 26 March 2015

Copyright: (c) 2015 Madar-Balakirski N, et al. This is an open-access article distributed under the terms of the Creative Commons Attribution License, which permits unrestricted use, distribution, and reproduction in any medium, provided the original author and source are credited.

\begin{abstract}
Objective: Monitoring of immune responses is essential in the care of immunosuppressed individuals, including rheumatic patients. Evaluation of cellular immunity is essential for confirming virus-specific effector cell functions, but it is poorly standardized, and suffers from technical limitations and inaccurate results. There is, therefore, a need for reliable techniques for assessing cell-mediated immunity. In this study we compared the cell-mediated immunity response to influenza vaccine between a population of Rheumatoid Arthritis (RA) patients and healthy subjects by three methods.
\end{abstract}

Methods: Trivalent influenza subunit vaccine was administered to $18 \mathrm{RA}$ patients who were taking diseasemodifying antirheumatic drugs and to 18 healthy controls. Peripheral Blood Mononuclear Cells (PBMCs) and sera were obtained immediately before and $\sim 28$ days after vaccination. Cell-mediated immunity responses to vaccination were evaluated by (1) flow cytometric analysis of IL-2/IFN- $\gamma$ production in activated CD4/CD8 T-cells, (2) enzymelinked immunosorbent assay for the analysis of IFN-y secretion, and (3) Granzyme B activity assay. Humoral response was evaluated by the hemagglutination inhibition assay.

Results: Vaccination induced a significant increase in PBMC IFN-y secretion and Granzyme B activity in the RA patients. Granzyme B activity also significantly increased in the controls, but there was no change in the levels of secreted IFN-y. No group differences in the frequencies of IFN- $/$ /L-2-producing activated CD4/CD8 T-cells were observed by flow cytometry. The geometric mean of hemagglutination inhibition antibody titers increased significantly for the $\mathrm{H} 1 \mathrm{~N} 1 / \mathrm{H} 3 \mathrm{~N} 2$ influenza strains in both groups.

Conclusions: Granzyme B activity assay was the only method to detect a significant cell-mediated immunity response in both groups while significant increase in IFN-y secretion was demonstrated only in RA patients. Flow cytometric analysis failed to show IL-2 and IFN- $y$ production in both groups. Currently available methods for measuring cellular responsiveness to influenza vaccination are inconsistent and limited in their ability to reflect acquired cellular immunity.

Keywords: Influenza; Inactivated influenza vaccine; Rheumatoid arthritis; Cell-mediated immunity

\section{Introduction}

Patients with autoimmune inflammatory rheumatic diseases, such as Rheumatic Arthritis (RA), have approximately twice the risk of infectious diseases compared with the normal population $[1,2]$. This increased susceptibility may be attributed to both disease-associated immunoregulatory imbalances, as well as to chronic use of immunosuppressive drugs [3]. One of the key mechanistic elements that leads to the increased vulnerability to infection is the inhibitory effect of disease-modifying antirheumatic drugs (DMARDS) on lymphocyte proliferation and the associated lymphocytopenia [4]. In addition, the lymphocyte counts of DMARD-treated patients may decrease to levels comparable with those of HIV patients [4].

Influenza is a contagious respiratory illness that is considered vaccine-preventable. Influenza vaccination was shown to reduce flurelated illness, hospitalizations and deaths in healthy populations, even when it afforded only moderate ( $60 \%)$ effectiveness [5]. The European League against Rheumatism (EULAR) recommends that the administration of inactivated influenza vaccination should be strongly considered for patients with rheumatic disease [6], even those who are treated with DMARDs or tumor-necrosis factor (TNF) inhibitors (with the exclusion of the B-cell depleting agent, rituximab). In most trials upon which the recommendations were based, efficacy was assessed by humoral responsiveness, which is currently considered the gold standard for seasonal influenza vaccines. However, in the case of virus-related illnesses, such as influenza, cellular immunity is another important pathway of response, since the ability of vaccines to induce cell-mediated immunity (CMI) is believed to be important in preventing severe disease and in enabling long-term immunological memory [7].

Both CD4 and CD8 T cells have been reported to play roles in the clearance of primary influenza virus infections. These cells can develop into highly active effector populations that migrate to the lung in large numbers. This CMI is characterized by stimulation of $\mathrm{T}$ cell cytokine production [8]. Several works have demonstrated that memory T cells 
may also provide long-term heterotypic protection against distinct Influenza virus [9-12]. The evaluation of CMI responses is, however, poorly standardized, requires specialized equipment and often suffers from technical limitations that eventually lead to inaccurate and inconsistent results.

We compared the CMI response to influenza vaccination as assessed by three common methods of measuring cellular immunity, flow cytometric analysis (FCA) of cytokine production, ELISA assay of IFN- $\gamma$ secretion and the Granzyme B activity assay in RA patients and healthy subjects and determined the agreement between those methods. We also concomitantly evaluated these patients' humoral immune responses.

\section{Materials and Methods}

\section{Study design}

The study was approved by the institutional medical ethics committee, and written informed consent was obtained from all participants. Eighteen consecutive RA patients and 18 apparently healthy volunteers were included in the study. The RA patients were being treated with conventional DMARDs, mostly Methotrexate (MTX). All subjects were examined twice: the first time at study entry, when they were vaccinated against seasonal influenza, and the second time 4 weeks later. The venous blood samples that were taken from all participants at both visits were used for measuring the complete blood count and the erythrocyte sedimentation rate, the anti-sera were used for measuring the antibody response by the Hemagglutination Inhibition (HI) test. The peripheral blood mononuclear cells (PBMCs) in these samples were separated. Information on the use of other medications, the influenza vaccination status in the previous year and any adverse reactions to the vaccination were recorded.

\section{Influenza vaccine}

VAXIGRIP $^{\circledast}$ (Inactivated Influenza Vaccine Trivalent Types A and B [Split Virion]) 2010/2011 season influenza vaccine, surface antigen, inactivated (Sanofi Pasteur SA, Lyon, France), including A/California/ 7/2009 (H1N1)-like virus, A/Perth/16/2009 (H3N2)-like virus and B/ Brisbane/60/2008-like virus, was administered to all participants during October-December, 2010.

\section{PBMC isolation}

The PBMCs were isolated from heparinized venous blood by density gradient centrifugation on Lymphoprep (Axis-Shield, Oslo, Norway) immediately after blood was drawn. The cells were then washed with phosphate-buffered saline (PBS), resuspended at 107 cells $/ \mathrm{ml}$ in fetal calf serum supplemented with $10 \%$ DMSO, transferred to pre-chilled $2 \mathrm{ml}$ cryovials and cryopreserved in a Nalgene freezing container providing a controlled freezing rate of $1^{\circ} \mathrm{C} / \mathrm{min}$ at $-80^{\circ} \mathrm{C}$. After 1-3 days, the cryovials were transferred to the vapor phase of a liquid nitrogen cryo tank. The cells were thawed by prompt resuspension at $1.5 \times 106 \mathrm{cell} / \mathrm{ml}$ in RPMI 1640 supplemented with heat-inactivated $10 \%$ fetal calf serum, penicillin $100 \mathrm{U} / \mathrm{ml}$, streptomycin $0.1 \mathrm{mg} / \mathrm{ml}$, and $2 \mathrm{mM}$ L-glutamine (Biological Industries, Israel), then counted before further treatment.

\section{Flow cytometric analysis (FCA)}

The PBMCs were cultured at $0.16 \mathrm{ml} /$ well in 96 -well plates $(0.5 \times$ 106 cells/well) and stimulated with an influenza antigen mix (final concentration of $1.5 \mu \mathrm{g} / \mathrm{ml}$ ), consisting of equal portions of $\beta$ propiolactone inactivated whole virus of $\mathrm{H} 1 \mathrm{~N} 1 \mathrm{~A} / \mathrm{Brisbane} / 59 / 2007$ (TGA 2008/81B), H3N2 A/Uruguay/716/2007 (A/Brisbane/10/2007like, NIBSC 8/124) and B B/Brisbane/60/2008 (TGA 2009/82B). Cells were cultured in medium only for negative control. Stimulation with 5 $\mu \mathrm{g} / \mathrm{ml}$ Staphylococcal enterotoxin B (SEB) (Sigma Aldrich, St. Louis, MO) served as a positive control. The PBMCs were incubated for 18 hours at $37^{\circ} \mathrm{C} 5 \% \mathrm{CO} 2$, and monensin (Sigma Aldrich) at a final concentration of $1 \mathrm{mM}$ was added for the final 16 hours. Following incubation, the cells were washed with cold phosphate-buffered solution (PBS) blocked in 10\% newborn calf serum (NBCS)/PBS, centrifuged and resuspended in blocking buffer containing phycoerythrin-Cy7 (PE-Cy7)-conjugated anti-CD69, GFP-conjugated anti-CD8 and allophycocyanin (APC)-eFluor 780-conjugated antiCD3 (eBioscience Inc., San Diego, CA) followed by 15 minutes of incubation on ice in the dark. The cells were then washed in blocking buffer and further incubated for 30 minutes in fixation/ permeabilization buffer (Foxp3 Staining Buffer Set, eBioscience Inc.) at $37^{\circ} \mathrm{C}$ in the dark. The cells were washed three times with permeabilization buffer, centrifuged and resuspended in 10\% NBCS/ permeabilization buffer containing APC-conjugated anti-IFN- $\gamma$ and PE-conjugated anti-IL2 (eBioscience Inc., San Diego, CA) for 30 minutes of incubation on ice in the dark. The cells were washed and analyzed on a BD FACSCanto II flow cytometer (Becton Dickinson). Lymphocytes were gated by forward and sideward scatters patterns, followed by gating of $\mathrm{CD} 3+/ \mathrm{CD} 8+$ (Figure 1A). Among those population CD69 \pm IFN- $\gamma \pm$ and CD69 \pm IL $2-\gamma \pm$ quadrants were set according to the unstimulated controls.

ELISA assay of IFN- $\gamma$ secretion from PBMCs: The PBMCs were cultured at $0.5 \mathrm{ml} /$ well in 48 -well plates $(1.8 \times 106$ cells/well $)$ and stimulated with an influenza antigen mix and SEB as described above. The secreted IFN- $\gamma$ concentration was measured by an ELISA kit (Peprotech, Rehovot, Israel) according to the manufacturer's instructions.

Granzyme B activity assay: The PBMCs were cultured at $0.5 \mathrm{ml} /$ well in 48-well plates (1.8 X 106 cells/well) and stimulated with an influenza antigen mix and SEB as described above. The cells were lysed in lysis buffer (150 mM NaCl, $15 \mathrm{mM}$ Tris, $1 \%$ Triton x100), then stored at $-760 \mathrm{C}$. Granzyme B activity was measured according to the protocol described by Gijzen et al. [13]. Briefly, frozen cell lysates were subjected to three freeze/thaw cycles to enable the release of Granzyme B. Recombinant Granzyme B standards (Enzo Life Sciences International, Inc., PA) and cell lysates were added in duplicate to a 96 -well plate $(20 \mu \mathrm{l} /$ well $)$. The reaction was started upon the addition of $80 \mu \mathrm{l}$ of substrate solution containing $400 \mu \mathrm{M}$ of Ac-IEPD-pNA substrate (Calbiochem, Darmstadt, Germany) in assay buffer (100 mM HEPES pH 7.5, 10\% (w/v), sucrose, 0.1\% (w/v) CHAPS, and $10 \mathrm{mM}$ DTT (Sigma Aldrich, Rehovot, Israel). The plate was sealed, covered and incubated in a dark humidified chamber at 370C for $20 \mathrm{~h}$. After incubation, the plate was read at $405 \mathrm{~nm}$. Granzyme B units were calculated using a 4 th order polynominal curve with a $\log$ (concentration)-log (absorbance) plot, and corrected for protein concentrations by the BCA protein assay (Thermo Scientific, IL).

Humoral response: The antibody response was measured by the HI test according to a standard WHO procedure as previously described [14]. The titer of an antiserum not showing any inhibition was 
Citation: Madar-Balakirski N, Arad U, Amir S, Mandelboim M, Mendelson E, et al. (2015) Measurement of Cellular Immunity to Influenza Vaccination in Rheumatoid Arthritis; Comparison of Three Assays. J Vaccines Vaccin 6: 278. doi:10.4172/2157-7560.1000278

Page 3 of 6

recorded as 1/10. Humoral response was defined as either a fourfold or greater rise in the titer of $\mathrm{HI}$ antibodies, or a rise from a non-protective baseline level $(<1 / 40)$ to $\geq 1 / 40)$. Geometric mean titers of antibodies were calculated to assess the immunity of the whole group.

Statistical analyses: Non-parametric tests were used for the analysis since most parameters were not normally distributed (based on the Shapiro-Wilk test). Parametric tests were performed for the log transformation of the parameters. Any two groups were compared using Student's t-test, the Mann-Whitney U non-parametric test and the Wilcoxon signed rank non-parametric test as applicable. Comparisons of the proportion of responders in the patient and control groups were assessed with Fisher's exact test. Statistical analyses were carried out using the IBM SPSS statistics system for Windows, release 20 .

\section{Results}

Characteristics of RA patients and healthy controls: The 36 study participants included 18 RA patients and 18 healthy controls. The male-to-female ratio was similar between the groups, while the mean age of the controls was significantly lower than that of the RA patients $(\mathrm{p}=0.014$, Table 1$)$. At the time of vaccination, all RA patients were being treated with at least one conventional DMARD (MTX, 10 patients, 55.5\%), prednisone (4 patients, 22.2\%) and Plaquenil (6 patients, $33.3 \%$ ).

\begin{tabular}{|l|l|l|}
\hline & $\begin{array}{l}\text { RA patients } \\
(\mathbf{n = 1 8 )}\end{array}$ & $\begin{array}{l}\text { Healthy controls } \\
(\mathbf{n = 1 8 )}\end{array}$ \\
\hline Age mean \pm SD in years & $60.4 \pm 13.8$ & $46.4 \pm 18.2$ \\
\hline Female:Male ratio & $1: 1.6$ & $1: 2$ \\
\hline $\begin{array}{l}\text { Duration of RA, y, median } \\
\text { (range) }\end{array}$ & $12(2-34)$ & \\
\hline DASb median (range) & $4.4(1.26-7.5)$ & \\
\hline Methotrexate , n (\%) & $10 / 18(55.5)$ & \\
\hline Prednisone, $n(\%)$ & $4 / 18(22.2)$ & \\
\hline Plaquenil , $n(\%)$ & $6 / 18(33.3)$ & \\
\hline aRA, rheumatoid arthritis; bDAS, disease activity score \\
\hline
\end{tabular}

Table 1: Baseline characteristics and disease parameters in RAa patients and healthy control subjects.

FCA of IL-2- and IFN- $\gamma$-producing CD4+/CD8+ cells: The FCA of CD4/CD8 $\mathrm{T}$ cell cytokine production was performed before and 4 weeks after the influenza vaccination (Figure 1). Influenza-specific cells were defined as the percentage of CD69+/IFN- $\gamma \gamma$ - and CD69+/ IL-2- producing cells within the total CD8 and CD4 T cell population in the antigen-stimulated cultures subtracted by those in the negative control cultures. In general, the mean percentage of cytokineproducing T cells (CD4 and CD8) was higher among the controls compared to the patient group, however not to a level of significance. Also, there was no significant difference in the frequencies of IFN- $\gamma /$ IL-2-producing activated CD4 or CD8 T-cells, either before and 4 weeks following influenza vaccination.

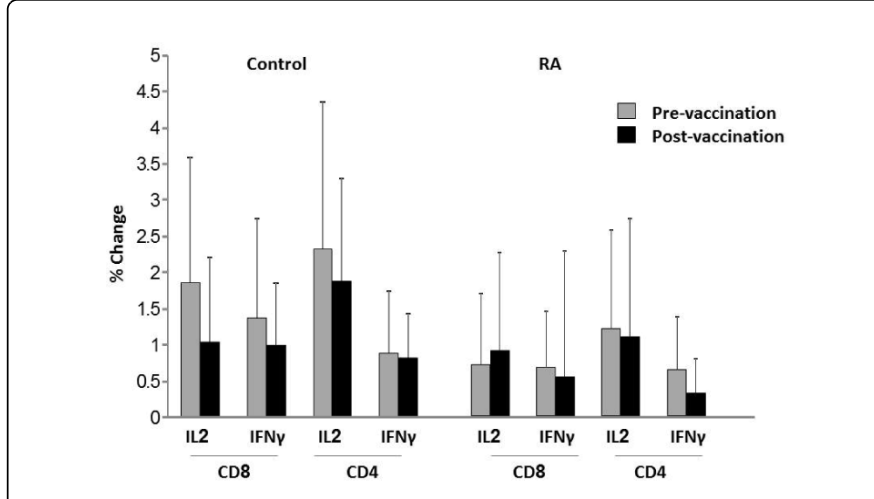

Figure 1: Flow cytometric analysis of of Il-2- and IFN $\gamma$-producing CD4+/CD8+ cells. The flow cytometric analysis of CD4/CD8 T-cell cytokine production was analyzed before and 4 weeks after influenza vaccination. Percent (\%) change was calculated as the percentage of $\mathrm{CD} 69+/ \mathrm{IFN}-\gamma$ - and CD69+/IL-2-producing cells within the total $\mathrm{CD} 8$ and $\mathrm{CD} 4 \mathrm{~T}$ cell population in the antigenstimulated cultures, subtracted by those in the negative control cultures. No difference in the frequencies of influenza specific activated cells was found in both groups, either before or 4 weeks following influenza vaccination (Columns, mean; Bars, SD). RA: Rheumatoid Arthritis.
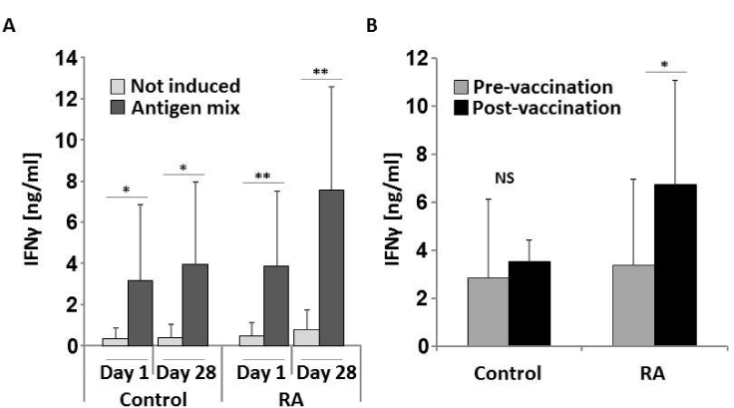

Figure 2: Analysis of IFN- $\gamma$ secretion by ELISA. IFN- $\gamma$ secretion levels were measured by ELISA in supernatants of peripheral blood mononuclear cells (PBMCs). (A) Stimulation with antigenic mixture (grey bars) led to significant increase in IFN- $\gamma$ secretion levels compared with untreated controls, in both groups, before and after immunization. (B) IFN- $\gamma$ net secretion levels of pre/post vaccination were calculated as the secreted IFN- $\gamma$ concentration levels in supernatants of antigen-stimulated cultures subtracted by those in the negative control cultures. Vaccination induced a significant increase in secretion levels in the rheumatoid arthritis patients but not in the healthy control group (Columns, mean; Bars, $\left.\mathrm{SD},{ }^{*} \mathrm{p} \leq 0.05,{ }^{* *} \mathrm{p} \leq 0.01\right)$. RA: Rheumatoid Arthritis.

IFN- $\gamma$ secretion from PBMCs: IFN- $\gamma$ secretion levels were measured by ELISA in the supernatants of PBMCs that were stimulated with either an influenza antigen mixture or with SEB, or left untreated. Stimulation of both groups with an antigenic mix led to a significant increase in secreted IFN- $\gamma$ concentrations from PBMCs compared with the untreated control secretion levels before as well as at four weeks post-vaccination (Figure 2A). However, when the pre- 
vaccination net secretion levels were compared to the post-vaccination ones (defined as the IFN- $\gamma$ concentrations in the stimulated cultures subtracted by those in the negative control cultures), the vaccination was found to induce a significant increase in PBMC IFN $\gamma$ secretion levels only in the RA patients, and not in the healthy controls (Figure 2B).

Granzyme B response: A Granzyme B standard curve was plotted as described in the Materials and Method section, with a standard line showing a squared correlation coefficient (R2) equal to 0.9997 in the range of 0-20 units (Figure 3A). Both groups showed a significant increase in Granzyme B activity levels in response to antigenic mixture stimulation at 4 weeks post-vaccination. Interestingly, no stimulationinduced increase in activity was observed on the day of vaccination (Figure 3B). A comparison of the pre- and post-vaccination net secretion levels (defined as the Granzyme B activity units/mg protein in the stimulated cultures subtracted by those in the negative control cultures) revealed a significant increase in Granzyme B activity in both the RA patients and healthy controls (Figure 3C).
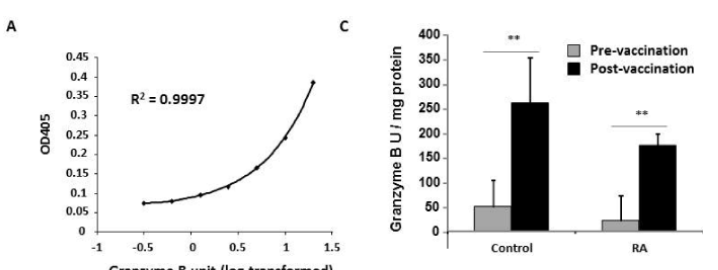

B

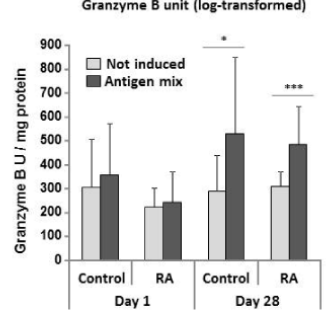

Figure 3: Granzyme B activity assay. Ganzyme B activity assays were measured in cultures of peripheral blood mononuclear cells (PBMCs) using a colorimetric substrate. A. The Granzyme B standard curve was plotted as described in the Materials and Method section. B. Stimulation with antigenic mixture in both groups led to an increase in Granzyme B activity compared with untreated controls 4 weeks post-vaccination. C. Comparison of preand post-vaccination net secretion levels (defined as the Granzyme $\mathrm{B}$ activity units/mg protein in the stimulated cultures subtracted by those in the negative control cultures) revealed a significant increase in Granzyme B activity in both rheumatoid arthritis patients and healthy controls (Columns, mean; Bars, standard deviation; $\left.{ }^{*} \mathrm{p} \leq 0.05,{ }^{* *} \mathrm{p} \leq 0.01,{ }^{* *} \mathrm{p}=0.068\right)$. RA: Rheumatoid Arthritis.

Immunogenicity of the vaccine: Both the RA patients and the controls displayed a significant increase in geometric mean titers of $\mathrm{HI}$ antibodies against two of the three vaccination serotypes at 4 weeks post-vaccination (Figure 4A). The geometric mean titers (GMTs) of A/ California/7/2009 (H1N1) increased from 12.97 to $86.21(\mathrm{p}=0.01)$ for the controls, and from 24.26 to $196.16(\mathrm{p}=0.005)$ for the RA group. The GMTS of A/Perth/16/2009 (H3N2) increased from 25.9 to 149.11 $(\mathrm{p}=0.005)$ for the controls, and from 18.18 to $211.72(\mathrm{p}=0.0004)$ for the RA patients. The GMTs of the third serotype, B/Brisbane/60/2008- like, increased for both groups (from 293.5 to 491.99 for the controls, and from 378.26 to 740.55 for the RA patients), but this increase did not reach a level of significance. There were no significant differences between the GMTs before and after vaccination between the patients and controls for each serotype examined.

Seroprotection and humoral response to vaccination: Seroprotection and response rates were calculated as described in the Materials and Methods section (Figures $4 \mathrm{~B}$ and 4C). The prevaccination seroprotection rates against the $\mathrm{B}$ strain were high for both groups tested $(94.4 \%$ and $100 \%$ for the controls and RA patients, respectively). This finding was in agreement with previous studies performed in our group [14-17].

The rate of responders to the B serotype was very low (16.67\% for both groups). The response rates to $\mathrm{H} 3 \mathrm{~N} 2$ was identical for both groups $(50 \%)$, while the response rate to the $\mathrm{H} 1 \mathrm{~N} 1$ was significantly higher in the patient group ( $77.8 \%$ Vs $38.9 \%$ for the controls, $\mathrm{p}=0.04$ ).

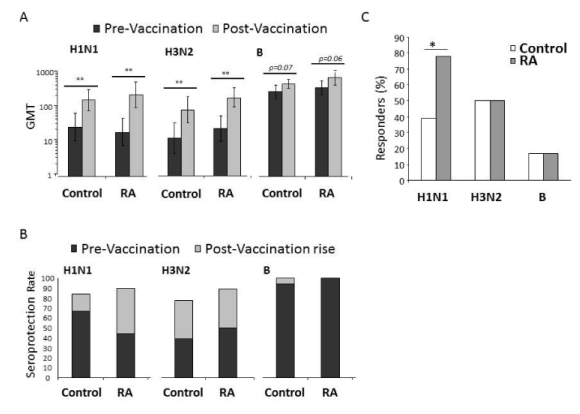

Figure 4: Humoral response to vaccination. Geometric mean titers (GMTs), seroprotection and response rates were calculated in sera of Rheumatoid Arthritis (RA) patients and healthy controls before and 4 weeks post-vaccination as described in the Materials and Methods section. A. There was a significant increase in titer for the H1N1 and H3N2 strains in both groups (Columns, geometric mean; Bars, standard deviation, $\left.{ }^{* *} \mathrm{p} \leq 0.01\right)$. B. Seroprotection was defined as an antibody titer of at least 1:40. C. Response rates for H1N1 were significantly higher in RA patients compared with healthy controls $\left({ }^{*} \mathrm{p} \leq 0.05\right)$.

\section{Discussion}

In the present study, we evaluated the cellular and humoral immune responses to seasonal influenza vaccination among patients with RA and compared them with the responses of a healthy control group. Humoral immunity was measured by the detection of influenza-specific antibody titer using the HI test pre- and postvaccination. A significant increase in the GMT of $\mathrm{HI}$ was demonstrated for the $\mathrm{H} 1 \mathrm{~N} 1$ and H3N2 influenza strains in both groups, and pre-vaccination protective titers for the B influenza stain were also observed for both groups. These results confirm a preserved humoral responsiveness in rheumatic patients, as had been shown by us in earlier studies [14-17].

We compared the extent of cell-mediated immunity by using three different methodologies, FCA of IFN- $\gamma /$ IL-2-producing activated CD4/CD8 T-cells, ELISA assay of IFN- $\gamma$ secretion and the Granzyme$B$ activity assay. Our results showed no significant change in the frequencies of IFN- $\gamma / \mathrm{IL}$-2-producing activated CD4 or CD8 T cells 
before and after vaccination in both groups when we used the FCA approach. Vaccination did induce a significant increase in PBMC IFN$\gamma$ secretion, as measured by ELISA, in the RA patient group but not in the control group. In contrast, there was a significant post-vaccination increase in Granzyme B activity in both groups, suggesting that this method is probably the most sensitive of the three in the evaluation of cellular response to seasonal influenza vaccine.

Taken together, these results reveal considerable inconsistency between three currently available methods for the measurement cellmediated immunity. Unexpectedly, FCA was the least sensitive among them. This is especially interesting since it is the one most commonly used in the clinical setting and also the most complicated one among the three since it requires expensive and complex instrumentation and demands considerable expertise to operate. Intracellular cytokine staining by FCA is one of the main assays currently used to monitor CMI responses. It is a powerful method that allows multiparametric, high-speed evaluation of unique subsets of cells that are otherwise difficult to distinguish [18]. Such detailed sub-setting of cells provides accurate analysis of immunological responses, especially when it is important to know what kind of response had been generated or in order to investigate the mechanism of the immunological response [19]. However, due to the rather low sensitivity of the technique, rare cell populations, such as antigen-specific $\mathrm{T}$ cells, are often not easily detected. Moreover, cytokine synthesis by cells is not constitutive, their intracellular concentrations are relatively low, and the kinetics of coexpression for different cytokines may vary. Thus, accurate detection may be difficult at relatively low frequencies of target cells [20]. Another possible explanation for the relatively low $\mathrm{T}$ cell response observed by FCA in immunocompromised patients is the choice of the type of vaccination. The most currently used influenza vaccines are the trivalent inactivated ones that consist of either a split virus or its surface glycoproteins, hemagglutinin and neuraminidase [21]. These vaccines induce a predominantly antibody response and lead to limited protection, especially in elderly or immunocompromised populations [22,23], presumably due to relatively poor cytotoxic $\mathrm{T}$ cell response. In contrast, the alternative live attenuated influenza vaccines were shown to induce a robust type 1 immunity response, characterized by IFN- $\gamma$ production and $\mathrm{T}$ cell recruitment [24]. However, these vaccines are not recommended by most published guidelines, including the EULAR guideline, for vaccination in adult patients with autoimmune inflammatory rheumatic diseases due to risk of infection [6].

Measurement of secreted IFN- $\gamma$ in PBMC supernatants by ELISA is another common technique routinely used to detect anti-viral T-cell response. This method is highly quantitative, it can measure a broad range of concentration, it is cost-effective and easy to operate. Indeed, our current ELISA results revealed that PBMC stimulation with an antigenic mix led to a significant increase in secreted IFN- $\gamma$ levels compared with the untreated PBMCs. However, no significant changes were found when we compared the pre- and post-vaccination values in the control group. IFN- $\gamma$ is a cytokine that plays a central role in promoting both innate and adaptive anti-viral immune responses by being released from Natural Killer (NK) and Natural Killer T (NKT) cells and by $\mathrm{CD} 4 \mathrm{Th} 1$ and $\mathrm{CD} 8$ cytotoxic $\mathrm{T}$ lymphocyte cells, respectively $[25,26]$. It is therefore possible that, at least in part, the measured IFN $-\gamma$ response was due to $\mathrm{NK}$ cells, as part of an innate immunity response.

Among the three methods tested in the current study, Granzyme B activity assay was the only one to detect an influenza-specific cellular immune response in both the RA and control groups. Granzymes are serine proteases that mediate caspase-cascade activation in virusinfected cells upon release from their effector cells, cytotoxic T cells and NK/NKT cells, and thus lead to the programmed cell death of their target [27]. The use of Granzyme B activity as a marker of cellmediated cytotoxicity has been evaluated before, and found to be specific, robust and in correlation with traditional measures of cytolytic activity $[28,29]$. In combination with antibody titers, Granzyme B activity levels were shown to predict vaccine efficacy in elderly populations $[13,30]$. They were also found to be the main contributor to cytotoxic $\mathrm{T}$ cell-mediated antiviral response in mice [31].

Our study has several limitations. The study groups were not matched for age, with the healthy controls being approximately 15 years younger than RA patients $(\mathrm{p}<0.05)$. Therefore, mismatching may have confounded our results, as such that older subjects have lower vaccine responses, but greater preexisting influenza exposure. Still, this mismatching does not influence the comparison between the different assays. A power calculation was not performed prior to the study since the variability of the assays was not known and blood samples from only 18 patients were available for the analyses. Hence, it is possible the study was underpowered to detect a difference in $\mathrm{T}$ cell responses by FCA or IFN- $\gamma$ ELISA. Other methods that we did not assess include ELISPOT analysis, a very sensitive assay that measures the numbers of cells that respond by secreting cytokines, and real-time PCR analysis, to quantify mRNA transcripts of response genes. These assays were not included since they are time consuming, expensive and require considerable expertise to be performed accurately and reproducibly, therefore limiting their usefulness in many scenarios.

In summary, we believe this to be the first study that compared the cellular response to influenza vaccination among RA patients as measured by three different methods. The results of this study confirm previous findings on the preserved humoral immune response of RA patients to influenza vaccination. The agreement between the cellular response to the humoral response was shown only by the Granzyme B activity assay but not by the ELISA or the FCA methods we had utilized to measure cellular immune responsiveness.

\section{Author's Contributions}

NMB participated in the design of the study, carried out the experiments, participated in the analysis and interpretation of data, performed the statistical analysis and drafted the manuscript. UA participated in the design of the study, helped to carry out the experiments, participated in the analysis and interpretation of data and helped to draft the manuscript. SA participated in the design of the study and in the collection of the samples. MM and EM performed and analyzed the hemagglutination inhibition assay. DC participated in the design and coordination of the study and helped to draft the manuscript. OA conceived of the study, participated in the design and coordination of the study and helped to draft the manuscript.

\section{Acknowledgement}

Esther Eshkol is thanked for editorial assistance.

\section{References}

1. Doran MF, Crowson CS, Pond GR, O'Fallon WM, Gabriel SE (2002) Frequency of infection in patients with rheumatoid arthritis compared with controls: a population-based study. Arthritis Rheum 46: 2287-2293. 
Citation: Madar-Balakirski N, Arad U, Amir S, Mandelboim M, Mendelson E, et al. (2015) Measurement of Cellular Immunity to Influenza Vaccination in Rheumatoid Arthritis; Comparison of Three Assays. J Vaccines Vaccin 6: 278. doi:10.4172/2157-7560.1000278

Page 6 of 6

2. Glück T, Müller-Ladner U (2008) Vaccination in patients with chronic rheumatic or autoimmune diseases. Clin Infect Dis 46: 1459-1465.

3. Listing J, Gerhold K, Zink A (2013) The risk of infections associated with rheumatoid arthritis, with its comorbidity and treatment. Rheumatology (Oxford) 52: 53-61.

4. Glück T, Kiefmann B, Grohmann M, Falk W, Straub RH, et al. (2005) Immune status and risk for infection in patients receiving chronic immunosuppressive therapy. J Rheumatol 32: 1473-1480.

5. Sieper J, van der Heijde D, Dougados M, Mease PJ, Maksymowych WP, et al. (2013) Efficacy and safety of adalimumab in patients with nonradiographic axial spondyloarthritis: results of a randomised placebocontrolled trial (ABILITY-1). Ann Rheum Dis 72: 815-822.

6. van Assen S, Agmon-Levin N, Elkayam O, Cervera R, Doran MF, et al (2011) EULAR recommendations for vaccination in adult patients with autoimmune inflammatory rheumatic diseases. Ann Rheum Dis 70: 414-422.

7. Thomas PG, Keating R, Hulse-Post DJ, Doherty PC (2006) Cell-mediated protection in influenza infection. Emerg Infect Dis 12: 48-54.

8. Su HC, Cousens LP, Fast LD, Slifka MK, Bungiro RD, et al. (1998) CD4+ and CD8+ T cell interactions in IFN-gamma and IL-4 responses to viral infections: requirements for IL-2. J Immunol 160: 5007-5017.

9. Kreijtz JH, Fouchier RA, Rimmelzwaan GF (2011) Immune responses to influenza virus infection. Virus Res 162: 19-30.

10. McKinstry KK1, Strutt TM, Swain SL (2011) Hallmarks of CD4 T cell immunity against influenza. J Intern Med 269: 507-518.

11. Richards KA, Topham D, Chaves FA, Sant AJ (2010) Cutting edge: CD4 $\mathrm{T}$ cells generated from encounter with seasonal influenza viruses and vaccines have broad protein specificity and can directly recognize naturally generated epitopes derived from the live pandemic $\mathrm{H} 1 \mathrm{~N} 1$ virus. J Immunol 185: 4998-5002.

12. Tu W, Mao H, Zheng J, Liu Y, Chiu SS, et al. (2010) Cytotoxic T lymphocytes established by seasonal human influenza cross-react against 2009 pandemic H1N1 influenza virus. J Virol 84: 6527-6535.

13. Gijzen K, Liu WM, Visontai I, Oftung F, van der Werf S, et al. (2010) Standardization and validation of assays determining cellular immune responses against influenza. Vaccine 28: 3416-3422.

14. Oren S, Mandelboim M, Braun-Moscovici Y, Paran D, Ablin J, et al (2008) Vaccination against influenza in patients with rheumatoid arthritis: the effect of rituximab on the humoral response. Ann Rheum Dis 67: 937-941.

15. Elkayam O (2006) Safety and efficacy of vaccination against influenza in patients with rheumatoid arthritis. Clin Dev Immunol 13: 349-351.

16. Elkayam O, Amir S, Mendelson E, Schwaber M, Grotto I, et al. (2011) Efficacy and safety of vaccination against pandemic 2009 influenza A (H1N1) virus among patients with rheumatic diseases. Arthritis Care Res (Hoboken) 63: 1062-1067.
17. Litinsky I, Balbir A, Zisman D, Mandelboim M, Mendelson E, et al. (2012) Vaccination against influenza in patients with systemic sclerosis. Clin Exp Rheumatol 30: S7-11.

18. Roederer M, Brenchley JM, Betts MR, De Rosa SC (2004) Flow cytometric analysis of vaccine responses: how many colors are enough? Clin Immunol 110: 199-205.

19. Querec TD, Akondy RS, Lee EK, Cao W, Nakaya HI, et al. (2009) Systems biology approach predicts immunogenicity of the yellow fever vaccine in humans. Nat Immunol 10: 116-125.

20. Sobti RS, Krishna A (2003) Advanced Flow Cytometry: Applications in Biological Research, Hardcover.

21. Nichol KL (2008) Efficacy and effectiveness of influenza vaccination. Vaccine 26 Suppl 4: D17-22.

22. Lambert ND, Ovsyannikova IG, Pankratz VS, Jacobson RM, Poland GA (2012) Understanding the immune response to seasonal influenza vaccination in older adults: a systems biology approach. Expert Rev Vaccines 11: 985-994.

23. McElhaney JE, Xie D, Hager WD, Barry MB, Wang Y, et al. (2006) T cell responses are better correlates of vaccine protection in the elderly. J Immunol 176: 6333-6339.

24. Lanthier PA, Huston GE, Moquin A, Eaton SM, Szaba FM, et al. (2011) Live attenuated influenza vaccine (LAIV) impacts innate and adaptive immune responses. Vaccine 29: 7849-7856.

25. Marshall NB, Swain SL (2011) Cytotoxic CD4 T cells in antiviral immunity. J Biomed Biotechnol 2011: 954602.

26. Schoenborn JR, Wilson CB (2007) Regulation of interferon-gamma during innate and adaptive immune responses. Adv Immunol 96: 41-101.

27. Darmon AJ, Nicholson DW, Bleackley RC (1995) Activation of the apoptotic protease CPP32 by cytotoxic T-cell-derived granzyme B. Nature 377: 446-448.

28. Ewen C, Kane KP, Shostak I, Griebel PJ, Bertram EM, et al. (2003) A novel cytotoxicity assay to evaluate antigen-specific CTL responses using a colorimetric substrate for Granzyme B. J Immunol Methods 276: 89-101.

29. Peters W, Brandl JR, Lindbloom JD, Martinez CJ, Scallan CD, et al. (2013) Oral administration of an adenovirus vector encoding both an avian influenza A hemagglutinin and a TLR3 ligand induces antigen specific granzyme $\mathrm{B}$ and IFN-gamma $\mathrm{T}$ cell responses in humans. Vaccine 31: 1752-1758.

30. McElhaney JE1, Ewen C, Zhou X, Kane KP, Xie D, et al. (2009) Granzyme B: Correlates with protection and enhanced CTL response to influenza vaccination in older adults. Vaccine 27: 2418-2425.

31. Lawrence CW, Ream RM, Braciale TJ (2005) Frequency, specificity, and sites of expansion of CD8+ T cells during primary pulmonary influenza virus infection. J Immunol 174: 5332-5340. 